\title{
Extensions and demonstrations of Hölder's inequality
}

\section{Fei $\operatorname{Yan}^{1 *}$ (D) and Qin Gao}

"Correspondence:

13705127132@163.com

${ }^{1}$ North China Electric Power

University, Baoding, P.R. China

Full list of author information is

available at the end of the article

\begin{abstract}
In this paper, we present some new extensions of Hölder's inequality and give a condition under which the equality holds. We also show that many existing inequalities related to the Hölder inequality are particular cases of the inequalities presented. At the same time, we further promote new inequalities based on the already introduced Hölder inequality.
\end{abstract}

MSC: Primary 26D15; secondary 39A13

Keywords: Hölder's inequality; Cauchy's inequality; Refinement; Generalization; Concave functions

\section{Introduction}

Let $a_{i j}>0,1 \leq i \leq m, 1 \leq j \leq n, p_{j}>1$, and $\sum_{j=1}^{n} p_{j}^{-1}=1$. The following Hölder inequality is well known:

$$
\sum_{i=1}^{m} \prod_{j=1}^{n} a_{i j} \leq \prod_{j=1}^{n}\left(\sum_{i=1}^{m} a_{i j}^{p_{j}}\right)^{\frac{1}{p_{j}}} .
$$

The integral form of the Hölder inequality is

$$
\int_{a}^{b}\left(\prod_{j=1}^{n} f_{j}(x)\right) d x \leq \prod_{j=1}^{n}\left(\int_{a}^{b} f_{j}^{p_{j}}(x) d x\right)^{1 / p_{j}} .
$$

In addition, from (1) the famous Cauchy inequality follows:

$$
\left(\sum_{i=1}^{n} x_{i} y_{i}\right)^{2} \leq\left(\sum_{i=1}^{n} x_{i}^{2}\right)\left(\sum_{i=1}^{n} y_{i}^{2}\right) .
$$

Analytic inequalities [1-12], especially Hölder's inequalities play an important role in mathematical analysis, harmonic analysis, functional analysis, and partial differential equations. It is precisely because of the importance of the Hölder inequality that more and more authors have invested in its research and have made a lot of optimization and advancement. In this paper, we describe the discrete and continuous forms of the Hölder inequality and its associated inferences.

(c) The Author(s) 2019. This article is distributed under the terms of the Creative Commons Attribution 4.0 International License (http://creativecommons.org/licenses/by/4.0/), which permits unrestricted use, distribution, and reproduction in any medium, provided you give appropriate credit to the original author(s) and the source, provide a link to the Creative Commons license, and indicate if changes were made. 
Yang's $[13,14]$ insights into inequalities have further led to several inferences. Qi's [15, 16] several integral inequalities explain the integral form of the inequality and further elaboration of the integral form of the Hölder inequality. Tian [17-20], Tian and Ha [21, 22], Tian, Ha, and Wang [23], Tian and Pedrycz [24], Kwon and Bae [25], Tian, Zhu and Cheung [26], and Zhao and Cheung [27] give a series of meaningful improvements, generalization, properties, and applications of Hölder's inequality. The papers in the references are of great guiding significance to the conception and promotion of this paper.

From the paper by Yang [14] we can get the following conclusions.

Let the function $h:(-\infty,+\infty) \rightarrow(0,+\infty)$ be defined as

$$
h(t)=\prod_{k=1}^{n}\left[\sum_{i=1}^{m}\left(\prod_{j=1}^{n} a_{i j}\right)^{1-t}\left(a_{i k}^{p_{k}}\right)^{t}\right]^{1 / p_{k}} .
$$

It is easy to see that $h \in C^{\infty}$ and (1) becomes

$$
h(0)=\sum_{i=1}^{m} \prod_{j=1}^{n} a_{i j} \leq \prod_{j=1}^{n}\left(\sum_{i=1}^{m} a_{i j}^{p_{j}}\right)^{\frac{1}{p_{j}}}=h(1) ;
$$

it is not difficult to see $h(0)=\sum_{i=1}^{m} \prod_{j=1}^{n} a_{i j}, h(1)=\prod_{j=1}^{n}\left(\sum_{i=1}^{m} a_{i j}^{p_{j}}\right)^{1 / p_{j}}$ under the conditions $p_{k}>1, k=1,2, \ldots, n$, and $\sum_{k=1}^{n} 1 / p_{k}=1$. From (2) it follows that

$$
h^{\prime}(t) \begin{cases}\geq 0, & t \geq 0 \\ \leq 0, & t \leq 0\end{cases}
$$

and the equation holds if and only if $t=0$ or $a_{i k}^{p_{k}} / \prod_{j=1}^{n} a_{i j}=a_{j k}^{p_{k}} / \prod_{j=1}^{n} a_{i j}$ for $1 \leq i, j \leq m$, $k=1,2, \ldots, n$.

From (2) and (3), it is natural to consider the function $h(t)$ more deeply. Similarly, we can define the function $g(t)$ related to the integral form of Hölder's inequality:

$$
g(t)=\prod_{k=1}^{n}\left[\int_{a}^{b}\left(\prod_{j=1}^{n} f_{j}(x)\right)^{1-t} f_{k}^{p_{k} t}(x) d x\right]^{1 / p_{k}}, \quad t \in \mathbb{R},
$$

where $f_{k}(x)>0, x \in[a, b], k=1,2, \ldots, n$, and $f_{k} \in L^{p_{k}}[a, b]$.

The integral expression of Hölder's inequality now becomes

$$
g(0)=\int_{a}^{b}\left(\prod_{k=1}^{n} f_{k}(x)\right) d x \leq \prod_{k=1}^{n}\left(\int_{a}^{b} f_{k}^{p_{k}}(x) d x\right)^{1 / p_{k}}=g(1) .
$$

As can be seen from the paper of Yang [14], (2) and (4) are generalized to the case $p_{j}>$ $1, \sum_{k=1}^{n} 1 / p_{k}=r$. We will show that $h_{r}(t)$ and $g_{r}(t)$ are concave functions on $\mathbb{R}$ and that $\min _{t \in \mathbb{R}} h_{r}(t)=h_{r}(0)$ and $\min _{t \in \mathbb{R}} g_{r}(t)=g_{r}(0)$; moreover, $h_{r}^{\prime}(t) t \geq 0$ and $g_{r}^{\prime}(t) t \geq 0$ for $t \in \mathbb{R}$. Therefore, we obtain refinements of (3) and (5):

$$
\begin{aligned}
& h_{r}(0)=h_{r}(1)-h_{r}^{\prime}(\tau), \quad \tau \in(0,1), \\
& g_{r}(0)=g_{r}(1)-g_{r}^{\prime}(s), \quad s \in(0,1),
\end{aligned}
$$


where $0 \leq h_{r}^{\prime}(\tau) \leq h_{r}^{\prime}(1)$ and $0 \leq g_{r}^{\prime}(s) \leq g_{r}^{\prime}(1)$. We also obtain conditions at which the equalities hold.

\section{Main results}

We begin this section with three lemmas, which will be used in the sequel.

From Yang [13] we get the following lemma.

Lemma 2.1 ([13]) If $A$ and $B$ are positive numbers, then

$$
(\ln A-\ln B)\left(A^{t}-B^{t}\right) \begin{cases}\geq 0, & t \geq 0 \\ \leq 0, & t \leq 0\end{cases}
$$

and the equality holds if and only if $(A-B) t=0$.

Lemma 2.2 ([14]) Let $r p_{k}>1, \sum_{k=1}^{n} \frac{1}{p_{k}}=r, a_{i j}>0,1 \leq i \leq m, 1 \leq j \leq n, b_{i}=\left(\prod_{j=1}^{n} a_{i j}\right)^{\frac{1}{r}}$, $d_{i k}=a_{i k}^{r p_{k}} / \prod_{j=1}^{n} a_{i j}, 1 \leq i \leq m, 1 \leq k \leq n$. Then

$$
\sum_{k=1}^{n} \frac{1}{p_{k}}\left(\sum_{i=1}^{m} b_{i} \ln d_{i k}\right)=0
$$

Proof By the definition of $b_{i}$ and $d_{i k}$ we have for $i=1,2, \ldots, m$ :

$$
\begin{aligned}
\sum_{k=1}^{n} \frac{1}{p_{k}} \ln d_{i k} & =\sum_{k=1}^{n} \frac{1}{p_{k}} \ln \left(\frac{a_{i k}^{r p_{k}}}{b_{i}^{r}}\right) \\
& =\sum_{k=1}^{n} r \ln a_{i k}-\sum_{k=1}^{n} \frac{r}{p_{k}} \ln b_{i} \\
& =r\left(\ln \left(\prod_{k=1}^{n} \frac{a_{i k}}{b_{i}^{r}}\right)\right) \\
& =0 .
\end{aligned}
$$

Therefore

$$
\sum_{k=1}^{n} \frac{1}{p_{k}}\left(\sum_{i=1}^{m} b_{i}^{r} \ln d_{i k}\right)=\sum_{i=1}^{m} b_{i}^{r}\left(\sum_{k=1}^{n} \frac{1}{p_{k}} \ln d_{i k}\right)=0 .
$$

Thus the proof of the lemma is completed.

Lemma 2.3 ([14]) Let $r p_{k}>1, \sum_{k=1}^{n} \frac{1}{p_{k}}=r, f_{k}(x)>0, F(x)=\left(\prod_{j=1}^{n} f_{j}(x)\right)^{\frac{1}{r}}, g_{k}(x)=f_{k}^{r p_{k}} / F^{r}(x)$, $x \in[a, b], k=1,2, \ldots, n$. Then

$$
\sum_{k=1}^{n} p_{k}^{-1} \ln g_{k}(x)=0, \quad x \in[a, b] .
$$


Proof After a simple operation, we have

$$
\begin{aligned}
\sum_{k=1}^{n} p_{k}^{-1} \ln g_{k}(x) & =\sum_{k=1}^{n} \frac{1}{p_{k}} \ln \frac{f_{k}^{r p_{k}}(x)}{F^{r}(x)} \\
& =\sum_{k=1}^{n} \frac{1}{p_{k}}\left[r p_{k} \ln f_{k}(x)-r \ln F(x)\right] \\
& =\sum_{k=1}^{n} \ln f_{k}(x)-\sum_{k=1}^{n} \frac{r}{p_{k}} \ln F(x) \\
& =r \ln \prod_{k=1}^{n} f_{k}(x)-r^{2} \ln F(x) \\
& =r \ln \frac{\prod_{k=1}^{n} f_{k}(x)}{F^{r}(x)} \\
& =0 .
\end{aligned}
$$

Thus the proof of the lemma is completed.

The main result of this paper is the following theorem.

As can be seen from Kwon and Bae [25], we can get the continuous function $h_{r}(t)$ proposed by Theorem 2.4. In this paper, we consider continuous variables $f(x, y)$ instead of discrete variables $a_{i j}$. At the same time, we will introduce the discrete form of $h_{r}(t)$ used in this paper by the continuous function.

Theorem 2.4 Let $X=(X, \mu)$ and $(Y, v)$ be positive measure spaces with $\mu(X)=1$ and $0<$ $v(Y)<\infty$. Let $f(x, y)$ be a real-valued bounded measurable function on $X \times Y$. Define the function $h_{r}(t): \mathbb{R} \rightarrow \mathbb{R}^{+}$by

$$
h_{r}(t)=\exp \left[\int_{X} \ln \left(\int_{Y} G(y) e^{t H(x, y)} d v(y)\right) d \mu(x)\right]
$$

where $G(y)=e^{\int_{X} f(x, y) d \mu(x)}$ and $H(x, y)=r f(x, y)-\int_{X} f(x, y) d \mu(x)$.

Then the discrete form of $h_{r}(t)$ can be further pushed out:

$$
h_{r}(t)=\prod_{k=1}^{n}\left[\sum_{i=1}^{n}\left(\prod_{j=1}^{m} X_{i j}\right)^{1-t}\left(X_{i k}^{r p_{k}}\right)^{t}\right]^{\frac{1}{p_{k}}},
$$

where $r p_{k}>1, \sum_{k=1}^{n} \frac{1}{p_{k}}=r, X_{i j}>0(1 \leq i \leq m, 1 \leq j \leq n)$, and $X_{i j}$ are measurable.

Proof Take $X, Y$ as two discrete spaces $X=1,2,3, \ldots, n, Y=1,2,3, \ldots, m$, and take a positive sequence $p_{j}$ satisfying $\sum_{j=1}^{n} \frac{1}{p_{j}}=r$. Set $\mu, v$ as

$$
\begin{aligned}
& \mu=\sum_{j=1}^{n} \frac{1}{p_{j}} \delta_{j}, \\
& v=\sum_{i=1}^{m} \delta_{i},
\end{aligned}
$$


where $\delta_{j}$ and $\delta_{i}$ denote the unit mass concentrated at $j$ and $i$, respectively. Then $\left(X, 2^{X}, \mu\right)$ and $\left(Y, 2^{Y}, v\right)$ are positive measure spaces with $\mu(X)=1$ and $0<v(Y)=m<\infty$. Here $2^{\{\cdot\}}$ means the set of all subsets of $\{\cdot\}$. Let

$$
e^{f(j, i)}=X_{i j}^{p_{j}}
$$

Then

$$
\begin{aligned}
\exp \left(\int_{X} f(x, y) d \mu(x)\right) & =\exp \left(\sum_{j=1}^{n} \frac{1}{p_{j}} \ln X_{i j}^{p_{j}}\right) \\
& =\exp \left(\sum_{j=1}^{n} \ln X_{i j}\right) \\
& =\exp \left(\ln \prod_{j=1}^{n} X_{i j}\right)=\prod_{j=1}^{n} X_{i j} .
\end{aligned}
$$

Therefore $h_{r}(t)$ has the expression

$$
\begin{aligned}
h_{r}(t) & =\exp \left[\int_{X} \ln \left(\int_{Y} G(y) e^{t H(x, y)} d \nu(y)\right) d \mu(x)\right] \\
& =\exp \left[\int_{X} \ln \left(\int_{Y} e_{X} f(x, y) d \mu(x) e^{r t f(x, y)-t \int_{X} f(x, y) d \mu(x)} d \nu(y)\right) d \mu(x)\right] \\
& =\exp \left[\int_{X} \ln \left(\int_{Y}\left(e^{\int_{X} f(x, y) d \mu(x)}\right)^{1-t}\left(e^{f(x, y)}\right)^{r t} d \nu(y)\right) d \mu(x)\right] \\
& =\exp \left[\int_{X} \ln \left(\int_{Y}\left(\prod_{j=1}^{n} X_{i j}\right)^{1-t}\left(X_{i k}^{r p_{k}}\right)^{t} d v(y)\right) d \mu(x)\right] \\
& =\exp \left[\int_{X} \ln \left(\sum_{i=1}^{m}\left(\prod_{j=1}^{n} X_{i j}\right)^{1-t}\left(X_{i k}^{r p_{k}}\right)^{t}\right) d \mu(x)\right] \\
& =\exp \left[\sum_{k=1}^{n} \frac{1}{p_{k}} \ln \left(\sum_{i=1}^{m}\left(\prod_{j=1}^{n} X_{i j}\right)^{1-t}\left(X_{i k}^{r p_{k}}\right)^{t}\right)\right] \\
& =\prod_{k=1}^{n}\left[\sum_{i=1}^{m}\left(\prod_{j=1}^{n} X_{i j}\right)^{1-t}\left(X_{i k}^{r p_{k}}\right)^{t}\right]^{\frac{1}{p_{k}}} \cdot
\end{aligned}
$$

Thus the proof of the theorem is completed.

Theorem 2.5 Let $a_{i j}>0(1 \leq i \leq m, 1 \leq j \leq n), r p_{k}>1, \sum_{k=1}^{n} 1 / p_{k}=r$. Define the positive function

$$
h_{r}(t)=\prod_{k=1}^{n}\left[\sum_{i=1}^{m}\left(\prod_{j=1}^{n} a_{i j}\right)^{1-t}\left(a_{i k}^{r p_{k}}\right)^{t}\right]^{1 / p_{k}}, \quad t \in \mathbb{R} .
$$


Then the function $h_{r}(t)$ defined by (8) is concave, that is, $h_{r}^{\prime \prime}(t) \geq 0$ for $t \in \mathbb{R}$, and the equality holds if and only if

$$
\frac{a_{i k}^{r p_{k}}}{\prod_{j=1}^{n} a_{i j}}=\frac{a_{j k}^{r p_{k}}}{\prod_{l=1}^{n} a_{j l}}, \quad 1 \leq i, j \leq m, k=1,2, \ldots, n
$$

In this case, $h_{r}(t)$ is a constant.

Proof It is easy to see from (2) that $h_{r}(t)$ can be expressed in the following equivalent form:

Let $b_{i}=\left(\prod_{j=1}^{n} a_{i j}\right)^{\frac{1}{r}}, d_{i k}=a_{i k}^{r p_{k}} / b_{i}^{r}$,

$$
h_{r}(t)=\prod_{k=1}^{n}\left[\sum_{i=1}^{m} b_{i}^{r}\left(\frac{a_{i k}^{r p_{k}}}{b_{i}^{r}}\right)^{t}\right]^{\frac{1}{p_{k}}} .
$$

Let $H_{r}(t)=\ln h_{r}(t)$,

$$
H_{r}(t)=\sum_{k=1}^{n} \frac{1}{p_{k}} \ln \left(\sum_{i=1}^{m} b_{i}^{r} d_{i k}^{t}\right)
$$

Then by Lemmas 2.1 and 2.2 we obtain

$$
\begin{aligned}
H_{r}^{\prime}(t)=\frac{h_{r}^{\prime}(t)}{h_{r}(t)} & =\sum_{k=1}^{n} \frac{1}{p_{k}} \frac{\sum_{i=1}^{m} b_{i}^{r} d_{i k}^{t} \ln d_{i k}}{\sum_{i=1}^{m} b_{i}^{r} d_{i k}^{t}} \\
& =\sum_{k=1}^{n} \frac{1}{p_{k}} \frac{\sum_{i=1}^{m} b_{i}^{r} d_{i k}^{t} \ln d_{i k}}{\sum_{i=1}^{m} b_{i}^{r} d_{i k}^{t}}-\sum_{k=1}^{n} \frac{1}{p_{k}} \frac{\sum_{i=1}^{m} b_{i}^{r} \ln d_{i k}}{\sum_{i=1}^{m} b_{i}^{r}} \\
& =\sum_{k=1}^{n} \frac{1}{p_{k}} \frac{\sum_{1 \leq i<j \leq m} b_{i}^{r} b_{j}^{r}\left(\ln d_{i k}-\ln d_{j k}\right)\left(d_{i k}^{t}-d_{j k}^{t}\right)}{\left(\sum_{i=1}^{m} b_{i}^{r}\right)\left(\sum_{i=1}^{m} b_{i}^{r} d_{i k}^{t}\right)} \quad \begin{cases}\geq 0, & t \geq 0, \\
\leq 0, & t \leq 0 .\end{cases}
\end{aligned}
$$

Therefore $H_{r}^{\prime}(t)=0$ if and only if $t=0$ or

$$
d_{i k}=d_{j k}, \quad 1 \leq i<j \leq m, k=1,2, \ldots, n .
$$

Similarly, by Lemmas 2.1 and 2.2 we obtain

$$
H_{r}^{\prime \prime}(t)=\sum_{k=1}^{n} \frac{1}{p_{k}} \frac{\left(\sum_{i=1}^{n} b_{i}^{r} d_{i k}^{t}\right)\left(\sum_{i=1}^{m} b_{i}^{r} d_{i k}^{t}\left(\ln d_{i k}\right)^{2}\right)-\left(\sum_{i=1}^{m} b_{i}^{r} d_{i k}^{t} \ln d_{i k}\right)^{2}}{\left(\sum_{i=1}^{m} b_{i}^{r} d_{i k}^{t}\right)^{2}} .
$$

It is easy to see that $H_{r}^{\prime \prime} \geq 0$ and the equality holds at some $t_{0}$ if and only if

$$
d_{i k}=d_{j k}, \quad 1 \leq i<j \leq m, k=1, \ldots, n .
$$

Since $h_{r}^{\prime}(t)=h_{r}(t) H_{r}^{\prime}(t)$, we have $h_{r}^{\prime \prime}(t)=h_{r}(t)\left[\left(H_{r}^{\prime}(t)\right)^{2}+H_{r}^{\prime \prime}(t)\right] \geq 0$, and $h_{r}^{\prime \prime}(t)=0$ if and only if $H_{r}^{\prime}(t)=0$ and $H_{r}^{\prime \prime}(t)=0$.

The proof of the theorem is completed. 
From our discussion we see that $h_{r}^{\prime \prime}(t) \geq 0$ and the equality holds at some $t_{0} \in \mathbb{R}$ if and only if (9) holds. In this case, $h_{r}^{\prime \prime}(t) \equiv 0$ and $h_{r}^{\prime}(t)=h_{r}^{\prime}(0)=$ const. Since, by by the proof, $h_{r}^{\prime}(t)=h_{r}(0) H_{r}^{\prime}(0)=0$, which yields $h_{r}(t)=h_{r}(0)=$ const, $t \in \mathbb{R}$.

Corollary 2.6 If (9) holds, then $h_{r}(t)=$ const. Otherwise, $h_{r}^{\prime \prime}(t)>0, t \in \mathbb{R}$, and $h_{r}^{\prime}(t) t>0$ for $t \neq 0$; in particular, for $0=t_{1}<t_{2}<\cdots<t_{N}=1$, we have

$$
\begin{aligned}
h_{r}(0) & =h_{r}\left(t_{1}\right)<h_{r}\left(t_{2}\right)<\cdots<h_{r}\left(t_{N}\right)=h_{r}(1), \\
h_{r}(0) & =\min _{t \in R} h_{r}(t)=\prod_{k=1}^{n}\left[\sum_{i=1}^{m} b_{i}^{r}\right]^{\frac{1}{p_{k}}}=\left(\sum_{i=1}^{m} b_{i}^{r}\right)^{r} \\
& =\left(\sum_{i=1}^{m} \prod_{j=1}^{n} a_{i j}\right)^{r} \leq h_{r}(1)=\prod_{k=1}^{n}\left[\sum_{i=1}^{m} a_{i k}^{r p_{k}}\right]^{\frac{1}{p_{k}}} .
\end{aligned}
$$

If $r=1$, then we get a refinement of (1),

$$
\sum_{i=1}^{m} \prod_{j=1}^{n} a_{i j} \leq \prod_{j=1}^{n}\left(\sum_{i=1}^{m} a_{i j}^{p_{j}}\right)^{\frac{1}{p_{j}}}
$$

Corollary 2.7 If (9) holds, then $h_{r}(t)=$ const. Otherwise,

$$
h_{r}(0)=h_{r}(1)-h_{r}^{\prime}(\tau), \quad \tau \in(0,1),
$$

and $0<h_{r}^{\prime}(\tau)<h_{r}^{\prime}(1)$, where

$$
h_{r}^{\prime}(1)=H_{r}^{\prime}(1) h_{r}(1)=\left(\sum_{k=1}^{n} \frac{1}{p_{k}} \frac{\sum_{1 \leq i<j \leq m} b_{i}^{r} b_{j}^{r}\left(\ln d_{i k}-\ln d_{j k}\right)\left(d_{i k}-d_{j k}\right)}{\left(\sum_{i=1}^{m} b_{i}^{r}\right)\left(\sum_{i=1}^{m} b_{i}^{r} d_{i k}\right)}\right) \prod_{j=1}^{n}\left(\sum_{j=1}^{n} a_{i j}^{p_{j}}\right)^{\frac{1}{p_{j}}} .
$$

Theorem 2.8 Let $f_{k}(x)>0, x \in[a, b], k=1,2, \ldots, n$, and $f_{k} \in L^{p_{k}}[a, b]$. Define the positive function

$$
g_{r}(t)=\prod_{k=1}^{n}\left[\int_{a}^{b}\left(\prod_{j=1}^{n} f_{j}(x)\right)^{1-t} f_{k}^{r p_{k} t}(x) d x\right]^{\frac{1}{p_{k}}}, \quad t \in \mathbb{R} .
$$

The function $g_{r}(t)$ defined by (10) is concave, that is, $g_{r}^{\prime \prime}(t) \geq 0$ for $t \in \mathbb{R}$, and the equality holds if and only if

$$
\frac{f_{k}^{r p_{k}}(x)}{\prod_{j=1}^{n} f_{j}(x)}=c_{k}=\text { const. }
$$

Proof It is known from Lemma 2.3 that if $F(x)=\left(\prod_{j=1}^{n} f_{j}(x)\right)^{\frac{1}{r}}$ and $g_{k}(x)=f_{k}^{r p_{k}}(x) / F^{r}(x)$, then

$$
g_{r}(t)=\prod_{k=1}^{n}\left[\int_{a}^{b} F^{r}(x) g_{k}^{t}(x) d x\right]^{\frac{1}{p_{k}}}
$$


Let $G_{r}(t)=\ln g_{r}(t)$. Then

$$
G_{r}(t)=\sum_{k=1}^{n} \frac{1}{p_{k}} \ln \left(\int_{a}^{b} F^{r}(x) g_{k}^{t}(x) d x\right)
$$

Then by Lemmas 2.1 and 2.3 we obtain

$$
\begin{aligned}
& G_{r}^{\prime}(t) \\
& \quad=\sum_{k=1}^{n} \frac{1}{p_{k}} \frac{\int_{a}^{b} F^{r}(x) g_{k}^{t}(x) \ln g_{k}(x) d x}{\int_{a}^{b} F^{r}(x) g_{k}^{t}(x) d x} \\
& \quad=\sum_{k=1}^{n} \frac{1}{p_{k}} \frac{\int_{a}^{b} F^{r}(x) g_{k}^{t}(x) \ln g_{k}(x) d x}{\int_{a}^{b} F^{r}(x) g_{k}^{t}(x) d x}-\frac{\int_{a}^{b} F^{r}(x)\left(\sum_{k=1}^{n} \frac{1}{p_{k}} \ln g_{k}(x)\right) d x}{\int_{a}^{b} F^{r}(x) d x} \\
& \quad=\sum_{k=1}^{n} \frac{1}{p_{k}}\left[\frac{\int_{a}^{b} F^{r}(x) g_{k}^{t}(x) \ln g_{k}(x) d x}{\int_{a}^{b} F^{r}(x) g_{k}^{t}(x) d x}-\frac{\int_{a}^{b} F^{r}(x) \ln g_{k}(x) d x}{\int_{a}^{b} F^{r}(x) d x}\right] \quad \begin{cases}\geq 0, & t \geq 0, \\
\leq 0, & t \leq 0 .\end{cases} \\
& \quad=\sum_{k=1}^{n} \frac{1}{2 p_{k}} \frac{\int_{a}^{b} \int_{a}^{b} F^{r}(x) F^{r}(y)\left(g_{k}^{t}(x)-g_{k}^{t}(y)\right)\left(\ln g_{k}(x)-\ln g_{k}(y)\right) d x d y}{\int_{k}(x) g_{k}^{t}(x) d x \int_{a}^{b} F^{r}(x) d x} \quad\left\{\begin{array}{l}
\geq x
\end{array}\right.
\end{aligned}
$$

Therefore $G_{r}^{\prime}(t)=0$ if and only if

$$
\frac{f_{k}^{r p_{k}}(x)}{\prod_{j=1}^{n} f_{j}(x)}=c_{k}=\text { const. }
$$

Similarly, by Lemmas 2.1 and 2.3 we obtain

$$
G_{r}^{\prime \prime}(t)=\sum_{k=1}^{n} \frac{1}{p_{k}} \frac{\int_{a}^{b} \int_{a}^{b} F^{r}(x) F^{r}(y) g_{k}^{t}(x) g_{k}^{t}(y)\left(\ln g_{k}(x)-\ln g_{k}(y)\right)^{2} d x d y}{2\left(\int_{a}^{b} F^{r}(x) g_{k}^{t}(x) d x\right)^{2}} \geq 0 .
$$

It is easy to see $G_{r}^{\prime \prime}(t) \geq 0$ and the equality holds at some $t_{0}$ if and only if

$$
\frac{f_{k}^{r p_{k}}(x)}{\prod_{j=1}^{n} f_{j}(x)}=c_{k}=\text { const. }
$$

Since $g_{r}^{\prime}(t)=g_{r}(t) G_{r}^{\prime}(t)$, we have $g_{r}^{\prime \prime}(t)=g_{r}(t)\left[\left(G_{r}^{\prime}(t)\right)^{2}+G_{r}^{\prime \prime}(t)\right] \geq 0$, and $g_{r}^{\prime \prime}(t)=0$ if and only if $G_{r}^{\prime}(t)=0$ and $G_{r}^{\prime \prime}(t)=0$.

Thus the proof of the theorem is completed.

Corollary 2.9 If $g_{k}(x)=$ const, then $g_{r}(t)=$ const. Otherwise, $g_{r}^{\prime \prime}(t)>0, t \in \mathbb{R}$, and $g_{r}^{\prime}(t) t>0$ for $t \neq 0$; in particular, for $0=t_{1}<t_{2}<\cdots<t_{N}=1$, we have

$$
\begin{aligned}
& g_{r}(0)=g_{r}\left(t_{1}\right)<g_{r}\left(t_{2}\right)<\cdots<g_{r}\left(t_{N}\right)=g_{r}(1), \\
& g_{r}(0)=\prod_{k=1}^{n}\left[\int_{a}^{b} F^{r}(x) d x\right]^{\frac{1}{p_{k}}} \leq g_{r}(1)=\prod_{k=1}^{n}\left[\int_{a}^{b} F^{r}(x) g_{k}(x) d x\right]^{\frac{1}{p_{k}}} .
\end{aligned}
$$


Corollary 2.10 If (11) holds, then $g_{r}(t)=$ const. Otherwise,

$$
g_{r}(0)=g_{r}(1)-g_{r}^{\prime}(s), \quad s \in(0,1),
$$

and $0<g_{r}^{\prime}(s)<g_{r}^{\prime}(1)$, where

$$
\begin{aligned}
g_{r}^{\prime}(1)= & G_{r}^{\prime}(1) g_{r}(1) \\
= & \sum_{k=1}^{n} \frac{1}{2 p_{k}} \frac{\int_{a}^{b} \int_{a}^{b} F^{r}(x) F^{r}(y)\left(g_{k}(x)-g_{k}(y)\right)\left(\ln g_{k}(x)-\ln g_{k}(y)\right) d x d y}{\int_{a}^{b} F^{r}(x) g_{k}(x) d x \int_{a}^{b} F^{r}(x) d x} \\
& \times \prod_{k=1}^{n}\left(\int_{a}^{b} f_{k}^{r p_{k}}(x) d x\right)^{\frac{1}{p_{k}}} .
\end{aligned}
$$

Next, we use the existing inequalities to derive the inequalities obtained in Kwon and Bae [25].

Theorem 2.11 Let $a_{i j}>0, p_{k}>1, \alpha_{k j} \in \mathbb{R}, 1 \leq i \leq m, 1 \leq j, k \leq n, \sum_{k=1}^{n} \frac{1}{p_{k}}=1$, and $\sum_{k=1}^{n} \alpha_{k j}=0$. Then

$$
\sum_{i=1}^{m} \prod_{j=1}^{n} a_{i j} \leq \prod_{k=1}^{n}\left(\sum_{i=1}^{m}\left(\prod_{j=1}^{n} a_{i j}^{1+p_{k} \alpha_{k j}}\right)\right)^{\frac{1}{p_{k}}}
$$

Moreover, for the integral form of this inequality, if $f_{j}(x)>0(j=1,2, \ldots, n), x \in[a, b],-\infty<$ $a<b<+\infty$, and $f_{j}(x) \in C[a, b]$, then

$$
\int_{a}^{b}\left(\prod_{j=1}^{n} f_{j}(x)\right) d x \leq \prod_{k=1}^{n}\left(\int_{a}^{b} \prod_{j=1}^{n} f_{j}^{1+p_{k} \alpha_{k j}}(x) d x\right)^{\frac{1}{p_{k}}}
$$

Proof From (2) we can see that $h(0)$ is the minimum point of $h(t)$.

Thus $h(0) \leq h(t)$, that is,

$$
\sum_{i=1}^{m} \prod_{j=1}^{n} a_{i j} \leq \prod_{k=1}^{n}\left[\sum_{i=1}^{m}\left(\prod_{j=1}^{n} a_{i j}\right)^{1-t}\left(a_{i k}^{p_{k}}\right)^{t}\right]^{\frac{1}{p_{k}}}
$$

Under the assumptions of (2), taking $t=-p_{k} \alpha_{k j}$ for $j \neq k$ and $t=\alpha_{k k} /\left(1-\frac{1}{p_{k}}\right)$ for $j=k$ with $\sum_{k=1}^{n} \alpha_{k j}=0$, we have

$$
\sum_{i=1}^{m} \prod_{j=1}^{n} a_{i j} \leq \prod_{k=1}^{n}\left(\sum_{i=1}^{m}\left(\prod_{j=1}^{n} a_{i j}^{1+p_{k} \alpha_{k j}}\right)\right)^{\frac{1}{p_{k}}}
$$

Similarly, the integral expression of Hölder's inequality now becomes $g(0) \leq g(t)$, that is,

$$
\int_{a}^{b}\left(\prod_{k=1}^{n} f_{k}(x)\right) d x \leq \prod_{k=1}^{n}\left(\int_{a}^{b}\left(\prod_{j=1}^{n} f_{j}(x)\right)^{1-t} f_{k}^{p_{k} t}(x) d x\right)^{\frac{1}{p_{k}}}, \quad t \in \mathbb{R} .
$$


Next, we make some changes to $g(t)$. Taking $t=-p_{k} \alpha_{k j}$ for $j \neq k$ and $t=\alpha_{k k} /\left(1-\frac{1}{p_{k}}\right)$ for $j=k$ with $\sum_{k=1}^{n} \alpha_{k j}=0$, we have

$$
\int_{a}^{b}\left(\prod_{j=1}^{n} f_{j}(x)\right) d x \leq \prod_{k=1}^{n}\left(\int_{a}^{b} \prod_{j=1}^{n} f_{j}^{1+p_{k} \alpha_{k j}}(x) d x\right)^{\frac{1}{p_{k}}} .
$$

Thus, the proof of the theorem is completed.

Similarly, we also consider the case $p_{j}>1, \sum_{k=1}^{n} \frac{1}{p_{k}}=r$.

Theorem 2.12 Let $a_{i j}>0, r p_{k}>1, \alpha_{k j} \in \mathbb{R}, 1 \leq i \leq m, 1 \leq j, k \leq n, \sum_{k=1}^{n} \frac{1}{p_{k}}=r$, and $\sum_{k=1}^{n} \alpha_{k j}=0$. Then

$$
\sum_{i=1}^{m} \prod_{j=1}^{n} a_{i j} \leq \prod_{k=1}^{n}\left(\sum_{i=1}^{m}\left(\prod_{j=1}^{n} a_{i j}^{1+r p_{k} \alpha_{k j}}\right)\right)^{\frac{1}{p_{k}}} .
$$

Moreover, for the integral form of this inequality, if $f_{j}(x)>0(j=1,2, \ldots, n), x \in[a, b],-\infty<$ $a<b<+\infty$, and $f_{j}(x) \in C[a, b]$, then

$$
\int_{a}^{b}\left(\prod_{j=1}^{n} f_{j}(x)\right) d x \leq \prod_{k=1}^{n}\left(\int_{a}^{b} \prod_{j=1}^{n} f_{j}^{1+r p_{k} \alpha_{k j}}(x) d x\right)^{\frac{1}{p_{k}}} .
$$

Proof From (8) we get that $h_{r}(0)$ is the minimum point of $h_{r}(t)$. Thus, $h_{r}(0) \leq h_{r}(t)$, that is,

$$
\sum_{i=1}^{m} \prod_{j=1}^{n} a_{i j} \leq \prod_{k=1}^{n}\left[\sum_{i=1}^{m}\left(\prod_{j=1}^{n} a_{i j}\right)^{1-t}\left(a_{i k}^{r p_{k}}\right)^{t}\right]^{\frac{1}{p_{k}}} .
$$

Under the assumptions of (8), taking $t=-r p_{k} \alpha_{k j}$ for $j \neq k$ and $t=\alpha_{k k} /\left(1-\frac{1}{r p_{k}}\right)$ for $j=k$ with $\sum_{k=1}^{n} \alpha_{k j}=0$, we have

$$
\sum_{i=1}^{m} \prod_{j=1}^{n} a_{i j} \leq \prod_{k=1}^{n}\left(\sum_{i=1}^{m}\left(\prod_{j=1}^{n} a_{i j}^{1+r p_{k} \alpha_{k j}}\right)\right)^{\frac{1}{p_{k}}} .
$$

Similarly, the integral expression of Hölder's inequality now becomes $g_{r}(0) \leq g_{r}(t)$, that is,

$$
\int_{a}^{b}\left(\prod_{k=1}^{n} f_{k}(x)\right) d x \leq \prod_{k=1}^{n}\left(\int_{a}^{b}\left(\prod_{j=1}^{n} f_{j}(x)\right)^{1-t} f_{k}^{r p_{k} t}(x) d x\right)^{\frac{1}{p_{k}}}, \quad t \in \mathbb{R} .
$$

Similarly to discrete $h_{r}(t)$, we also make some changes to the continuous $g_{r}(t)$. Taking $t=-r p_{k} \alpha_{k j}$ for $j \neq k$ and $t=\alpha_{k k} /\left(1-\frac{1}{p_{k}}\right)$ for $j=k$ with $\sum_{k=1}^{n} \alpha_{k j}=0$, we have

$$
\int_{a}^{b}\left(\prod_{j=1}^{n} f_{j}(x)\right) d x \leq \prod_{k=1}^{n}\left(\int_{a}^{b} \prod_{j=1}^{n} f_{j}^{1+r p_{k} \alpha_{k j}}(x) d x\right)^{\frac{1}{p_{k}}} .
$$

Thus the proof of the theorem is completed. 


\section{Conclusion}

As is well known, the Hölder inequality and its derivative theorems play an important role in mathematical analysis. In this paper, we have presented a wider range of discrete function $h_{r}(t)$ and continuous function $g_{r}(t)$ based on the existing discrete function $h(t)$ and continuous function $g(t)$. In addition, we give a proof and application of $h_{r}(t)$ and $g_{r}(t)$, and, finally, by using the results obtained we get an extension of Hölder's inequality to the new inequality. In the future research, we will continue to explore other derived inequalities of Hölder's inequality.

\section{Acknowledgements}

The authors would like to express their sincere thanks to the anonymous referees for their great efforts to improve this paper.

\section{Funding}

This work was supported by the Fundamental Research Funds for the Central Universities (No. 2015ZD29) and the Higher School Science Research Funds of Hebei Province of China (No. Z2015137).

\section{Competing interests}

The authors declare that they have no competing interests.

\section{Authors' contributions}

The authors read and approved the final manuscript.

\section{Author details}

${ }^{1}$ North China Electric Power University, Baoding, P.R. China. ${ }^{2}$ College of Science and Technology, North China Electric Power University, Baoding, P.R. China.

\section{Publisher's Note}

Springer Nature remains neutral with regard to jurisdictional claims in published maps and institutional affiliations.

Received: 6 September 2018 Accepted: 29 March 2019 Published online: 08 April 2019

\section{References}

1. Qi, F.: An algebraic inequality. J. Inequal. Pure Appl. Math. 2(1), Article ID 13 (2001)

2. Qi, F., Chen, S.-X.: Complete monotonicity of the logarithmic mean. Math. Inequal. Appl. 10(4), 799-804 (2007)

3. Qi, F., Li, W.-H.: A unified proof of several inequalities and some new inequalities involving Neuman-Sándor mean. Miskolc Math. Notes 15(2), 665-675 (2014)

4. Qi, F., Luo, Q.-M.: A simple proof of monotonicity for extended mean values. J. Math. Anal. Appl. 224(2), 356-359 (1998)

5. Tian, J.-F.: Note on common fixed point theorems in fuzzy metric spaces using the CLRg property. Fuzzy Sets Syst. https://doi.org/10.1016/j.fss.2019.01.018

6. Yang, Zh.-H., Tian, J.-F.: Monotonicity rules for the ratio of two Laplace transforms with applications. J. Math. Anal. Appl. 470(2), 821-845 (2019)

7. Yang, Zh.-H., Tian, J.-F.: A comparison theorem for two divided differences and applications to special functions. J. Math. Anal. Appl. 464(1), 580-595 (2018)

8. Yang, Zh.-H., Tian, J.-F.: An accurate approximation formula for gamma function. J. Inequal. Appl. $2018,56(2018)$

9. Yang, Zh.-H., Tian, J.-F.: Two asymptotic expansions for gamma function developed by Windschitl's formula. Open Math. 16, 1048-1060 (2018)

10. Yang, Zh.-H., Tian, J.-F.: Sharp inequalities for the generalized elliptic integrals of the first kind. Ramanujan J. 48(1), 91-116 (2019)

11. Yang, Zh.-H., Tian, J.-F.: A class of completely mixed monotonic functions involving the gamma function with applications. Proc. Am. Math. Soc. 146(11), 4707-4721 (2018)

12. Zhang, S.-Q., Qi, F.: Some integral inequalities. J. Henan Norm. Univ. Nat. Sci. 27(1), 88-89 (1997)

13. Yang, X.-J.: A note on Hölder inequality. Appl. Math. Comput. 134, 319-322 (2003)

14. Yang, X.-J.: Hölder's inequality. Appl. Math. Lett. 16, 897-903 (2003)

15. Qi, F.: Several integral inequalities. J. Inequal. Pure Appl. Math. 1(2), Article ID 19 (2000)

16. Qi, F., Luo, Q.-M.: Refinements and extensions of an inequality. Math. Inform. Q. 9(1), 23-25 (1999)

17. Tian, J.-F.: New property of a generalized Hölder's inequality and its applications. Inf. Sci. 288, 45-54 (2014)

18. Tian, J.-F.: Reversed version of a generalized sharp Hölder's inequality and its applications. Inf. Sci. 201, 61-69 (2012)

19. Tian, J.-F.: Inequalities and mathematical properties of uncertain variables. Fuzzy Optim. Decis. Mak. 10(4), 357-368 (2011)

20. Tian, J.-F.: Triple diamond-alpha integral and Hölder-type inequalities. J. Inequal. Appl. 2018, 111 (2018)

21. Tian, J.-F., Ha, M.-H.: Properties of generalized sharp Hölder's inequalities. J. Math. Inequal. 11(2), $511-525$ (2017)

22. Tian, J.-F., Ha, M.-H.: Extensions of Hölder's inequality via pseudo-integral. Math. Probl. Eng. 2018, Article ID 4080619 (2018) 
23. Tian, J.-F., Ha, M.-H., Wang, Ch.: Improvements of generalized Hölder's inequalities and their applications. J. Math. Inequal. 12(2), 459-471 (2018)

24. Tian, J.-F., Pedrycz, W.: New refinements of generalized Hölder's inequality and their applications. Math. Inequal. Appl. 19(3), 805-822 (2016)

25. Kwon, E.-G., Bae, E.-K.: On a continuous form of Hölder inequality. J. Math. Anal. Appl. 343, 585-592 (2008)

26. Tian, J.-F., Zhu, Y.-R., Cheung, W.-S.: N-tuple Diamond-Alpha integral and inequalities on time scales. Rev. R. Acad. Cienc. Exactas Fís. Nat., Ser. A Mat. https://doi.org/10.1007/s13398-018-0609-6

27. Zhao, C.-J., Cheung, W.-S.: Hölder's reverse inequality and its application. Publ. Inst. Math. 99(113), 211-216 (2016)

Submit your manuscript to a SpringerOpen ${ }^{\circ}$ journal and benefit from:

- Convenient online submission

- Rigorous peer review

- Open access: articles freely available online

- High visibility within the field

- Retaining the copyright to your article

Submit your next manuscript at $\gg$ springeropen.com 